\title{
Changes in $\gamma$-Tubulin Protein Distribution in Zebrafish (Danio rerio) Oocytes and the Early Cleavage-Stage Embryo
}

\author{
Jianxiong Liu ${ }^{1,2}$ and Charles A. Lessman ${ }^{1,3}$ \\ ${ }^{1}$ Department of Biology, The University of Memphis, Memphis, TN 38152, USA \\ ${ }^{2}$ Department of Physiology, University of Tennessee-Memphis, Memphis, TN 38163, USA \\ ${ }^{3}$ Department of Biological Sciences, The University of Memphis, 223 Life Science Building, Memphis, TN 38152-3560, USA
}

Correspondence should be addressed to Charles A. Lessman; clessman@memphis.edu

Received 1 October 2012; Accepted 23 October 2012

Academic Editors: O. Bossinger and M. Lardelli

Copyright (C) 2013 J. Liu and C. A. Lessman. This is an open access article distributed under the Creative Commons Attribution License, which permits unrestricted use, distribution, and reproduction in any medium, provided the original work is properly cited.

\begin{abstract}
We investigated the distribution of $\gamma$-tubulin in zebrafish oocytes and embryos using epifluorescent or confocal microscopy and $\gamma$-tubulin antibodies. During meiotic maturation of zebrafish oocytes, $\gamma$-tubulin begins redistribution from oocyte ooplasm and cortex to the future blastodisc region at the animal pole. In activated eggs, $\gamma$-tubulin was uniformly distributed in the enlarging blastodisc with label emanating from the yolk cell. In newly fertilized eggs, $\gamma$-tubulin was evenly distributed in blastomere cytoplasm, with the presence of pronuclei but initially lacking discernable centrosomes. During early cleavage, especially at the eight-cell stage, striking arc-shaped/rings (A/R) of putative centrosomes were detected. Decreasing $\gamma$-tubulin was seen in yolk cells while early cleavage blastomeres had strong cytoplasmic label along with obvious A/R arrays. In addition, we found the orientation of the $A / R$ array and nuclear division alternated by about 90 degrees for each cell cycle along with alternation of punctate and $A / R$ arrays.
\end{abstract}

\section{Introduction}

The microtubule cytoskeleton is essential for a variety of cellular processes, including cell movement, organelle transport, and cell division. Moreover, in oocytes and early embryos, microtubules have been implicated in localization of important embryonic determinants such as bicoid mRNA in Drosophila [1] and Vg1 mRNA in Xenopus [2] as well as trafficking cell components such as $\beta$-catenin in cleaving zebrafish embryos [3]. Recently, imaging of cytoskeleton in live zebrafish embryos has been described and the field reviewed [4]. The highly ordered microtubular array found in typical eukaryotic cells is organized by the microtubule-organizing center (MTOC). MTOCs organize microtubules by initiating noncovalent assembly of $\alpha-/ \beta$ tubulin heterodimers, anchoring them at their minus ends, and facilitating microtubule extension at the rapidly growing plus ends [5]. The morphology, subcellular localization, and molecular makeup of MTOCs vary across different species and different cell types within single species. The major MTOC in proliferating animal cells is the centrosome. Because of its central role in many essential aspects of cell physiology, both in interphase and during cell division, intense research activity has been carried out to characterize centrosomes [6-8]. Recently a review, describing centrosome and basal body research in zebrafish, has been published that enumerates the advantages of the zebrafish model in the study of MTOCs [9].

In most animal cells, centrosomes are composed of a pair of centrioles, surrounded by an amorphous cloud of electron-dense material, the pericentriolar material (PCM) [10]. An extensively characterized PCM protein required for MT nucleation is $\gamma$-tubulin. In a number of species, including Drosophila and Xenopus, $\gamma$-tubulin forms a macromolecular complex (called the $\gamma$-tubulin ring complex, $\gamma$ TuRC), with a characteristic ring structure ( $25 \mathrm{~nm}$ in diameter), together with a variety of associated proteins [11-15]. Hundreds of $\gamma$ TuRCs tether to the centrosome scaffold, to serve as 
the site of origin for MTs [15], and are required for spindle assembly and progression through mitosis [16-20]. Thus, the centrosome is the primary site for microtubule nucleation, and the centrosomal $\gamma$ TuRC is thought to be necessary for anchoring of MTs minus ends to centrosomes [2124] Using immunofluorescence and GFP reporter construct techniques, $\gamma$-tubulin was shown to be associated with centrosomes dynamically in mitotic cells, being massively recruited at prophase and released at anaphase-telophase. This accumulation in mitotic centrosomes is dramatic during the first embryonic divisions [25]. Moreover, fluorescence measurements suggest that the amount of antigenic $\gamma$-tubulin increases during mitosis and that the total amount of $\gamma$ tubulin in the spindle is larger than the amount of $\gamma$-tubulin in the spindle poles [26]. The cell stage distribution of $\gamma$-tubulin varies between animal cells of different species and between cells of different tissues within the same species. The function of the soluble cytoplasmic $\gamma$-tubulin remains unclear, and very little is known about how the $\gamma$ TuRC is assembled and tethered in the centrosome. Moreover, the redistribution of centrosomal proteins to specific sites of the cell is poorly understood, as are the mechanisms controlling microtubule nucleation within the living cell.

The zebrafish (Danio rerio), a small tropical freshwater teleost, has emerged as a model for cell and developmental biology because of its high fecundity, short generation time, and rapid development of the externally fertilized and translucent embryos [27]. We previously revealed the presence of soluble tubulin pools in zebrafish oocytes, which were associated with large-molecular-weight complexes, including $\alpha$-, $\gamma$-tubulin and other proteins. These ovarian tubulin complexes were sequestered and maintained in a temporary "oligomeric" state and appear to be relatively stable storage forms of soluble tubulins, which subsequently may play important roles in microtubule dynamics in zebrafish oogenesis and embryogenesis [28].

Early embryonic, maternally regulated vertebrate cell cycles (i.e., cleavage) differ from somatic cell cycles. Early embryonic cells have rapid synchronous cell cycles with alternating $\mathrm{M}$ (mitosis) and S (DNA synthesis) phases and lack G (gap/growth) phases. The rapid repeated cleavages result in cells with successively smaller volumes. Zebrafish embryogenesis begins with 10 metasynchronous mitotic cycles [29], and the genome is silenced until the ninth cleavage cycle [30]. These early divisions are remarkably fast, with 15 minutes per cycle [31]. Subsequently, the cycles slow gradually, leading to a transition from maternal to zygotic control of the cell cycle in cycle 10 , indicating that meiosis, mitosis, and many other essential processes required for embryonic development are regulated by maternal factors until this stage [32]. Since only 15 minutes are allotted for (1) the replication of the entire genome, (2) the assembly of the spindles with centrosomes, and (3) the segregation of chromosomes for each early cell cycle, it is remarkable that about two thousand centrosomes are calculated (i.e., a pair per dividing cell) to be assembled in less than 3 hours. Moreover, this stage of development relies primarily on maternally supplied materials and does not depend on zygotic gene expression until after the midblastula transition [30]. So all the components needed to build thousands of centrosomes should be preformed and laid down in the oocyte by the mother. Thus maternal regulation is a key feature of early development with many components necessary for embryogenesis already stored in the oocyte. This suggests that the oocyte, egg, and early embryo should be excellent sources of centrosome and spindle components for biochemical analysis. In a previous paper, using biochemical techniques, we demonstrated the presence of $\gamma$-tubulin along with $\alpha$-tubulin in large, soluble complexes ( 2 MDa) in zebrafish oocytes, eggs, and early embryos [28]. In this present study, using epifluorescent and confocal microscopy with monoclonal antibody against $\gamma$-tubulin (GTU-88), we aimed to reveal the cellular distribution of zebrafish $\gamma$-tubulin in oocytes and during early embryogenesis.

\section{Results}

2.1. Cellular Distribution of $\gamma$-Tubulin in Zebrafish Oocytes. To visualize the cellular distribution of $\gamma$-tubulin in zebrafish oocytes, the $\gamma$-tubulin proteins were detected specifically with immunofluorescence staining using monoclonal antibody GTU-88, followed by Alexa green-conjugated secondary antibody. Figure 1 illustrates the localization of $\gamma$-tubulin in fully grown oocytes during the time course of oocyte maturation in the latter induced with $17 \alpha-20 \beta-$ dihydroxyprogesterone (DHP) in vitro. In fully grown, immature oocytes (i.e., prophase I of meiosis), under lower magnification of confocal microscopy, $\gamma$-tubulin signals were detected uniformly in the ooplasm and around the cortex area (Figure 1(a)). Upon maturation represented by the oocytes treated with DHP, much of the $\gamma$-tubulin remains uniformly distributed, but some signal starts to accumulate in the future blastodisc region as the oocyte proceeds to metaphase II of meiosis (Figures 1(b) and 1(c), representing 2 hours and 4 hours of DHP treatment, resp.). Furthermore, naturally matured and oviposited eggs activated artificially with water to release the cells from the metaphase II block revealed that $\gamma$-tubulin proteins were found uniformly distributed in the enlarged blastodisc area, with decreased staining in the yolk cell (Figure 1(d)). The images obtained from higher magnification revealed discreet $\gamma$-tubulin foci and diffuse labeling in cytoplasm of immature oocytes (Figure $1(\mathrm{e})$, arrow heads). Formation of the animal pole blastodisc (Figures 1(f) and 1(g)) and clearing of the opaque ooplasm are markers for meiotic maturation in the live cells, as is the dissolution of the oocyte nucleus or germinal vesicle (GV; Figures $1(\mathrm{j})$ and $1(\mathrm{k}))$ [33]. The $\gamma$-tubulin continued to stream from the yolk cell and into the blastomeres at the $4-8$ cell stage as seen in confocal optical section (Figure 1(1)). Also apparent is the presence of diffuse $\gamma$-tubulin in the cytoplasm of the cleaving blastomeres along with intensely labeled putative centrosomes, while the yolk cell has markedly reduced label (Figure 1(l)). These data were strongly consistent with our in situ hybridization results [34], which revealed that $\gamma$-tubulin mRNA was diffusely distributed along the oocyte cortex area, but became localized to the blastodisc of the animal pole in mature oocytes (i.e., eggs) and blastomeres in dividing embryos. 


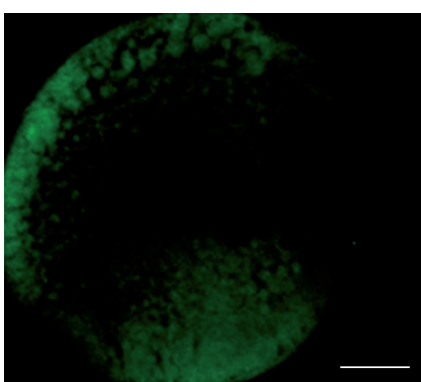

(a)

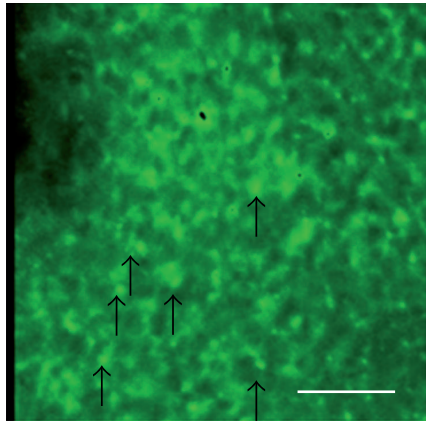

(e)

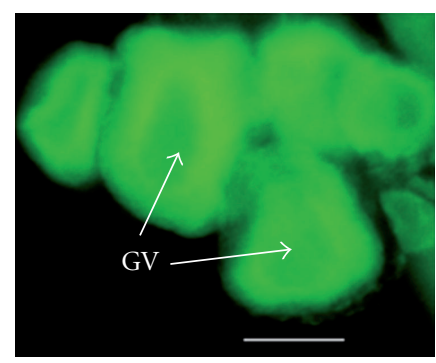

(i)

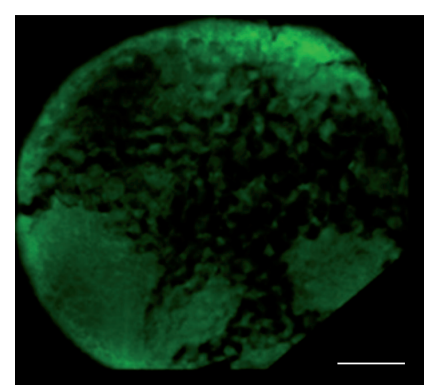

(b)

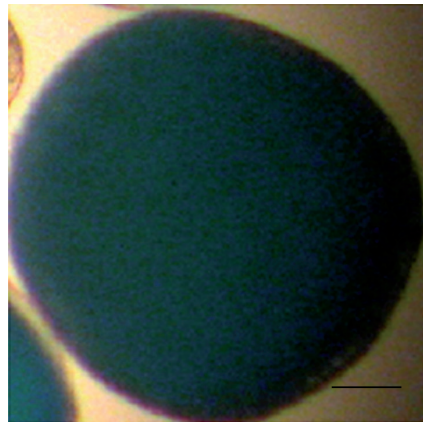

(f)

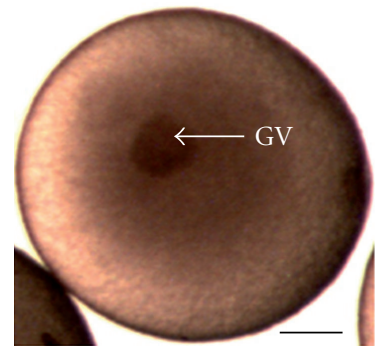

(j)

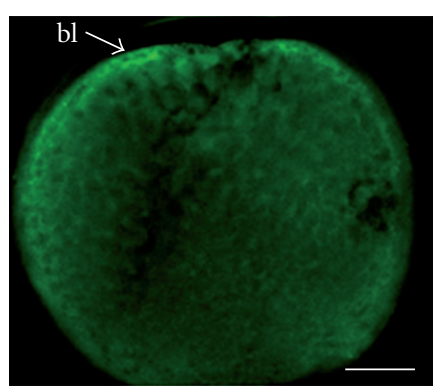

(c)

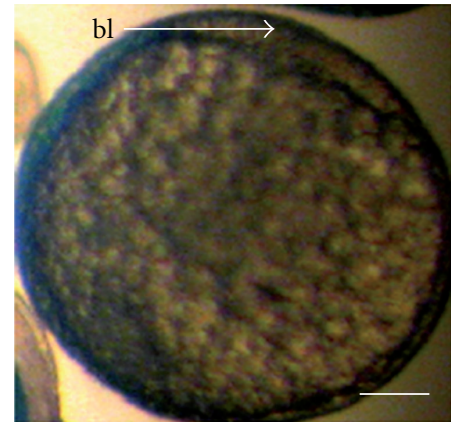

(g)

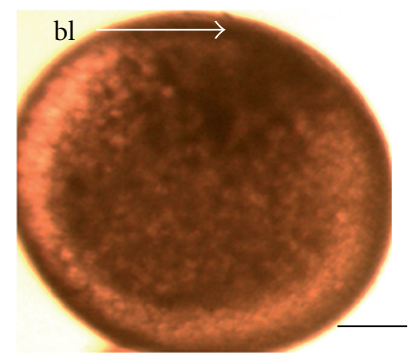

(k)

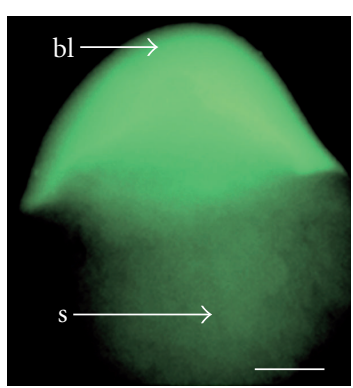

(d)

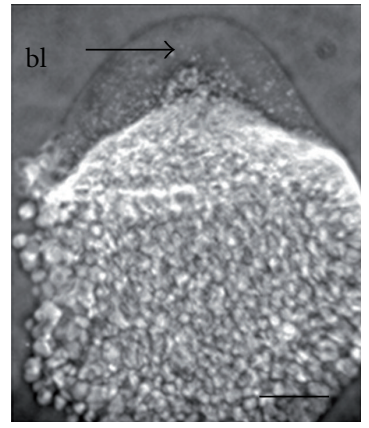

(h)

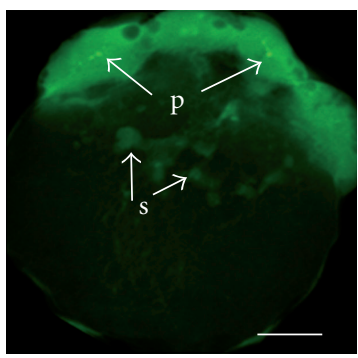

(1)

FIGURE 1: $\gamma$-Tubulin in oocytes is generally dispersed as punctate label that becomes increasingly localized to the animal pole during oocyte maturation, egg activation, and early cleavage; bars equal $100 \mu \mathrm{m}$. (a)-(e), (i) and (l) labeled for $\gamma$-tubulin with GTU-88; (f)-(h), (j) and (k) brightfield. (a) fully grown late stage III [72] immature oocyte, optical section with confocal microscope. (b) Stage IV oocyte (maturing) after $2 \mathrm{hr}$ of 17 $\alpha$-, 20 $\beta$-dihydroxyprogesterone (DHP), optical section with confocal microscope. (c) Stage V oocyte (i.e., mature egg) after $4 \mathrm{hr}$ of DHP treatment, optical section with confocal microscope; forming blastodisc (bl). (d) Artificially activated egg imaged with an epifluorescence microscope; enlarged blastodisc (bl), streams (s) of label emanating from yolk cell. (e) Late stage III immature oocyte, optical section with confocal microscope using higher magnification, arrow heads point to discreet $\gamma$-tubulin foci. (f) Live stage IV oocyte after $2 \mathrm{hr}$ of DHP treatment under bright field (DHP-treated oocytes usually remain opaque from 90-120 minutes then begin to clear). (g) Live stage V oocyte after $4 \mathrm{hr}$ of DHP treatment under brightfield (note the naturally cleared ooplasm and distinct blastodisc forming, bl); (h) artificially activated egg under brightfield (same as (d)). (i) Previtellogenic stages I and II oocytes with large central nucleus (germinal vesicle, GV) show $\gamma$-tubulin in ooplasm. (j) Stage IV oocyte after $2 \mathrm{hr}$ of DHP artificially cleared with $5 \%$ acetic acid, revealing the migrating GV. (k) Stage V oocyte (i.e., mature egg) after $4 \mathrm{hr}$ of DHP artificially cleared with 5\% acetic acid revealing the absence of the GV (i.e., undergone dissolution or GVD), and the blastodisc (bl) begins to form at the animal pole. (l) 8-cell embryo labeled for $\gamma$-tubulin, optical section with confocal microscope, revealing within the blastomeres (top) punctate centrosome structures (p), diffuse label in cytoplasm of blastomeres and wispy streams (s) of label extending from the yolk cell. "bl" in (c), (d), (g), (h), and (k) represents blastodisc region; all bars represent $100 \mu \mathrm{m}$.

\subsection{Cellular Distribution of $\gamma$-Tubulin in Zebrafish Embryos.} Figure 2 demonstrates the cellular distribution of $\gamma$-tubulin in early cleavage embryos. In newly fertilized 1-cell embryos, $\gamma$-tubulin was found to be uniformly distributed (stained green with GTU-88) in embryonic cytoplasm, with distinguishable $4^{\prime}$,6-diamidino-2-phenylindole (DAPI) stained pronuclei (arrow head, pseudocolored red). No obvious centrosomal structure was detected at this stage (Figure 2(a)). After approximately 30 minutes, when the embryo finished the first mitotic cycle and became a 2-cell embryo, clusters of putative centrosomal material with intense GTU-88 label were assembled around nuclei (Figure 2(b), arrow heads mark nuclei). The blastomere cytoplasm was also diffusely stained with GTU-88 around the brightly stained clusters of 

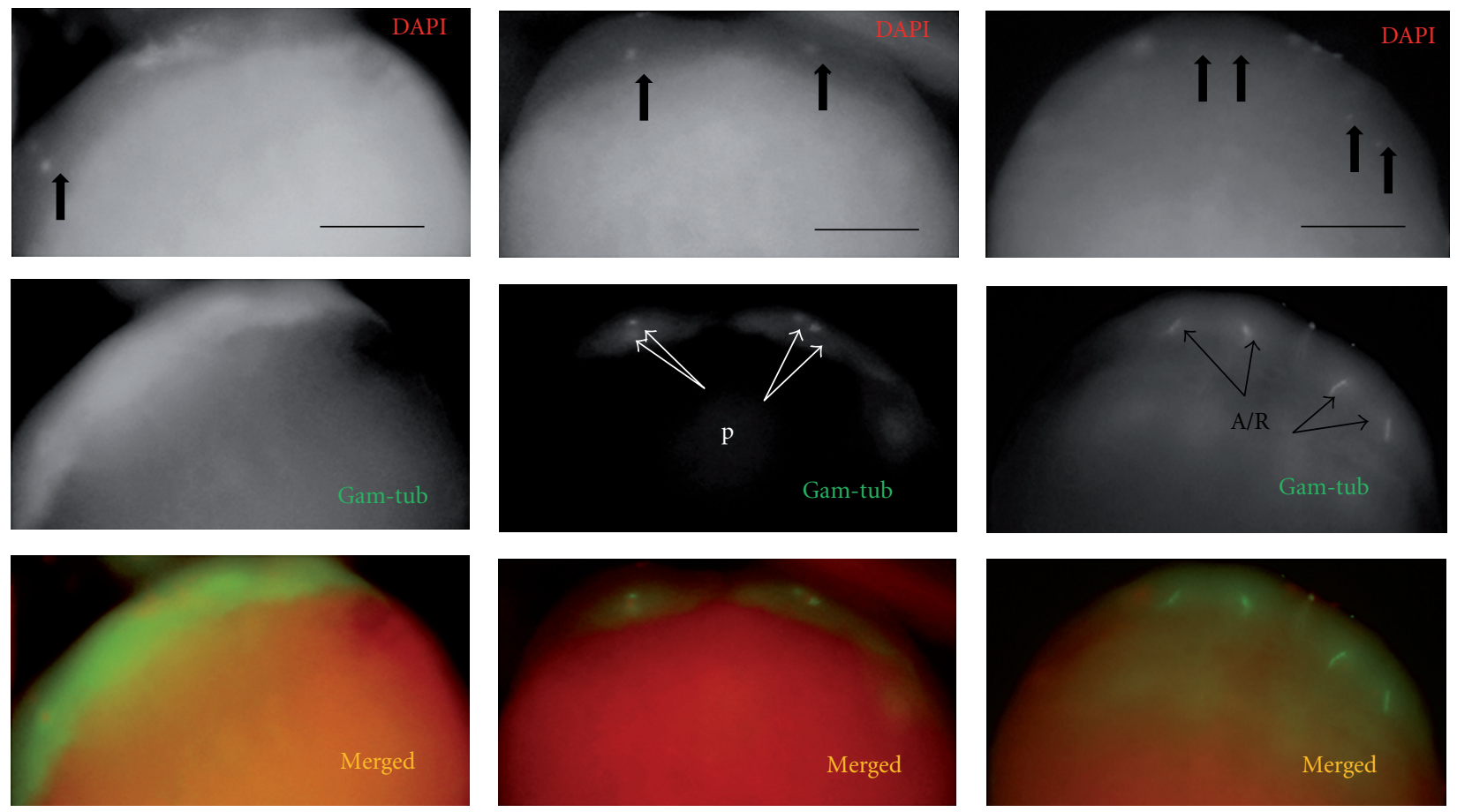

(a)

(b)

(c)

FIGURE 2: Cellular distribution of $\gamma$-tubulin in early cleavage embryos imaged with an epifluorescence microscope. (a), (b), and (c) represent 1-cell, 2-cell, and 4-cell (becoming 8 cell) stage zebrafish embryos, respectively. (Top) Nuclei within blastomeres were stained with DAPI (red, arrow heads point to nuclei); yolk cell nonspecifically stains with DAPI. (Middle) $\gamma$-tubulins were stained with GTU-88 (green); bar equals $100 \mu \mathrm{m} ; \gamma$-Tubulin is predominately found in blastomeres compared to yolk cells. (Bottom) "Merged" panels are a composite of both DAPI and GTU-88 staining. (a) Fertilization-stage embryo with a pronucleus (arrow) but no centrosomes only diffuse $\gamma$-tubulin staining in the blastodisc cytoplasm. (b) 2-cell stage with distinct punctate $\gamma$-tubulin clusters (p) in putative centrosomes polarized on opposite sides of the nuclei. (c) 4-cell embryo dividing into 8-cell stage; one bank of cells (upper) is in focus, while the other bank (lower) is not. The curvilinear or arc-shaped/ring (A/R) arrays of $\gamma$-tubulin (i.e., putative centrosome arrays) are particularly striking and extensive at this stage (c).

$\gamma$-tubulin in putative centrosomal structures (Figure 2(b)). In most 4 to 8 -cell embryos, centrosomes appeared with prominent curvilinear-array shapes (Figure 2(c)) associated with late mitotic phases (i.e., telophase). These interesting arc-shaped accumulations of $\gamma$-tubulin varied in length from about 10 to $25 \mu \mathrm{m}$ or more.

In cleavage and blastula staged embryos, the $\gamma$-tubulin positive structures are varied in morphology ranging from punctuate foci $(\mathrm{P})$ to "arc-shaped/ring" (A/R) structures (Figure 3). Interphase nuclei tended to have arc-shaped/ring clusters of $\gamma$-tubulin positive structures (Figures 3(a)-3(c)). Figure 3(d) shows an early blastula with both foci and arcshaped/rings in different blastomeres. When double-labeled with DAPI and GTU-88, the mitotic blastomeres tended to have foci at the spindle poles enriched in $\gamma$-tubulin (i.e., conventional centrosomes; Figures 3(e) and 3(f)). In addition, the blastomeres all contained significant levels of diffuse $\gamma$-tubulin compared to the yolk cell suggesting continued partitioning of this protein.

The results support our previous hypothesis [28] that maternal, soluble tubulin, including $\gamma$-tubulin, is accumulated and maintained in a temporary "oligomeric" form that is composed of large molecular weight complexes residing in the $40,000 \times \mathrm{g}$ supernatant pool during zebrafish oogenesis. In addition, this pool of maternal tubulin complexes would be subsequently sequestered and reallocated into many structures (e.g., centrosomes) and incorporated into embryonic MT networks in ensuing embryogenesis [28].

\subsection{Nuclei and Centrosomal Orientation during Zebrafish} Early Embryogenesis. Since embryonic centrosomes were undetectable in the 1-cell stage then suddenly appeared during the first mitotic division, we wanted to further investigate their cellular distribution in dividing blastomeres in later embryonic stages. The image data demonstrated that the centrosome arrays alternated from curvilinear or arc-shaped to punctate in one direction, then from punctate to arcshaped or curvilinear in another direction, while the nuclear division orientation alternated after each cell division (Figure 4). The striking curvilinear arrays of $\gamma$-tubulin appeared in blastomeres in late mitosis (i.e., telophase) during early cleavage (Figures 4(a), 4(b), and 4(e)). The schematic diagrams, indicating nuclei (red dots) and centrosomes (green lines or dots) for each embryonic stage, were derived from representative micrographs. 


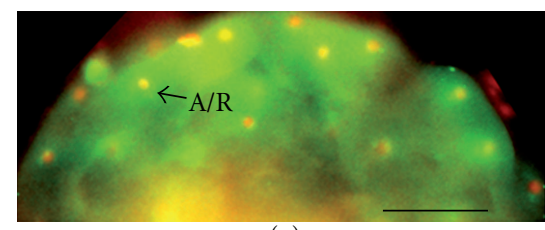

(a)

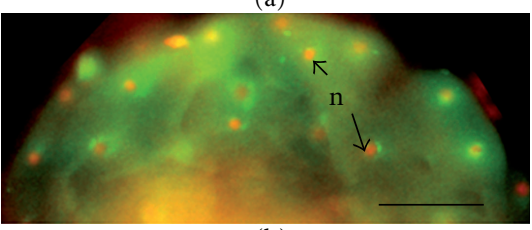

(b)

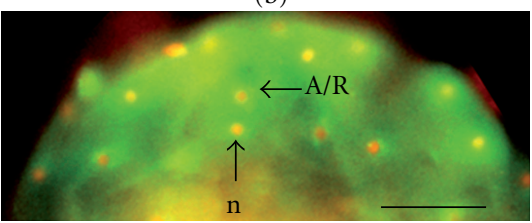

(c)

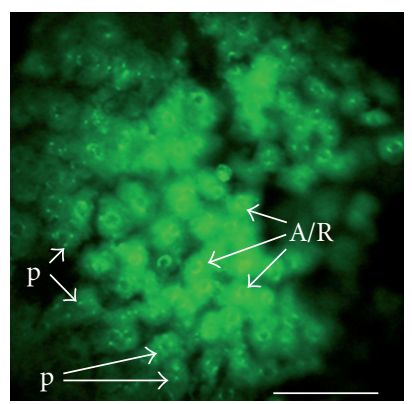

(d)

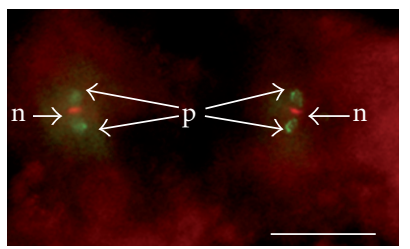

(e)

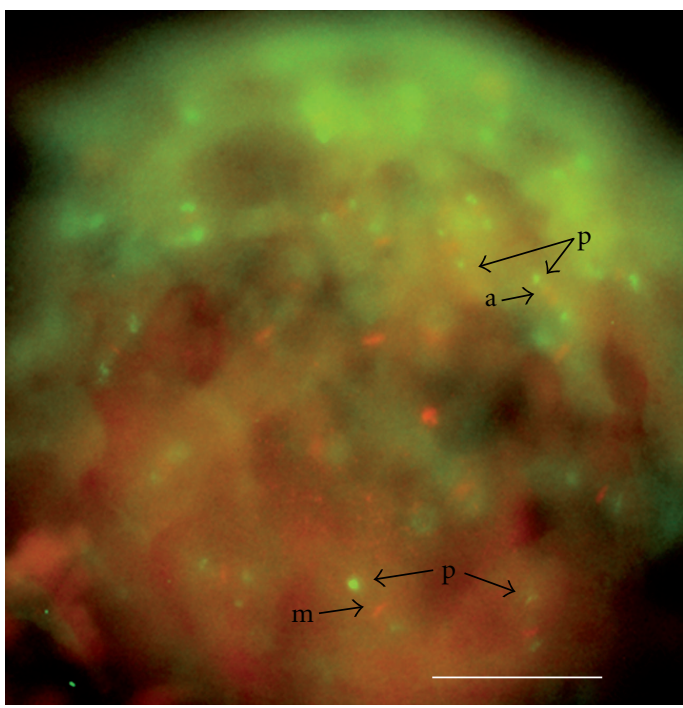

(f)

FIGURE 3: During cleavage, $\gamma$-tubulin is found in various structures including punctate foci (P) and arc-shaped/rings (A/R) revealed by GTU88 staining as well as diffuse presumably soluble, $\gamma$-tubulin in the cytoplasm of blastomeres. (a)-(c) Serial optical sections through a 32 -cell stage embryo revealing the extent of the A/R structures (arrows) surrounding interphase nuclei (n); DAPI (red), GTU-88 (green), bar equals $100 \mu \mathrm{m}$. (d) Early blastula showing both punctate foci (P) and arc-shaped/ring (A/R) structures stained with GTU-88, presumably associated with asynchronous blastomeres at different stages of the cell cycle; bar equals $100 \mu \mathrm{m}$. (e) 2-cell stage with punctate foci (P) of $\gamma$-tubulin (green) at the spindle poles of metaphase blastomeres, nuclei/chromosomes (n) stained with DAPI (red); bar equals $100 \mu \mathrm{m}$. (f) Early blastula with predominately mitotic blastomeres showing GTU-88 staining (green) in punctate foci (P) at the spindle poles of DAPI-stained (red) mitotic figures in metaphase (m) and anaphase (a); bar equals $100 \mu \mathrm{m}$.

\section{Discussion}

3.1. $\gamma$-Tubulin and Centrosomes during Oogenesis and Embryogenesis. The results presented here, demonstrating a lack of typical $\gamma$-tubulin-enriched centrosomes in zebrafish oocytes, unfertilized eggs, and water-activated eggs, are consistent with findings for other species [35, 36]. These findings further suggest that eccentric spindle placement for polar body formation in zebrafish does not require $\gamma$ tubulin enriched centrosomes, since these were not seen in oocytes or eggs. Typical centrosomes consist of a centriole pair surrounded by pericentriolar material (PCM). Previous work suggested that centrioles are required to organize PCM to form a structurally stable organelle. The PCM contains a matrix of 12-15 nm fibers termed the "centromatrix" [37]. The "centromatrix" is tightly associated with the centrioles and directs the recruitment of other centrosomal components including ring-shaped complexes containing $\gamma$-tubulin $[23,37]$. Thus, its centriolar stoichiometry may affect the rate of PCM recruitment or the retention of PCM at the centrosome by activating components for assembly or by affecting their turnover. In addition to control by a centriolar structural element, limiting levels of PCM components also influence centrosome size. SAS-4 is a centriole-associated component whose amount dictates centrosome size [38]. Such a regulatory agent may be responsible for the increased size of the $\gamma$-tubulin arrays seen here in early cleaving zebrafish embryos. An effect of PCM component limitation is expected because centrosomes get smaller when the ratio of cytoplasmic volume to centriole number is reduced during embryonic development [38].

Xenopus oocytes and eggs lack recognizable centrioles or centrosomes [39, 40]. The maternal centrosomes of Xenopus oocytes are inactivated early in stage I of oogenesis [40]. Despite lacking centrioles or definitive centrosomes, Xenopus eggs contain a substantial pool of centrosome components $[41,42]$ including $\gamma$-tubulin [19]. Using confocal immunofluorescence microscopy, $\gamma$-tubulin is found to be apparent surrounding the germinal vesicle $(\mathrm{GV})$ of stage VI Xenopus oocytes [43]. It is also concentrated in the cortex of stage VI oocytes and it is bound to spindle microtubules only after full elongation of the meiotic spindle then $\gamma$-tubulin became heavily concentrated at the spindle poles [44]. In the animal hemisphere, $\boldsymbol{\gamma}$-tubulin is evenly distributed as small cortical foci.

Centrosomes are not an essential component in the formation of the metaphase spindle during meiotic maturation of horse oocytes, but they can be introduced from the spermatozoon or donor cell and are necessary for the organization of normal embryonic development [45]. Centrosomes of the horse oocyte reorganize themselves from the beginning of GV stage to leave only PCM of $\gamma$-tubulin surrounding both poles of the MI and MII stage spindles [45].

In unfertilized mouse oocytes, meiosis is arrested in the second meiotic metaphase. $\gamma$-tubulin is found to be evenly distributed in both the cytoplasm and the germinal vesicle in the fully grown germinal vesicle $(\mathrm{GV})$ stage oocytes. After GVBD, $\gamma$-tubulin dots were localized in both the cytoplasm 


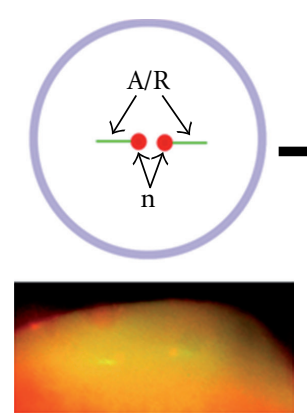

(a)

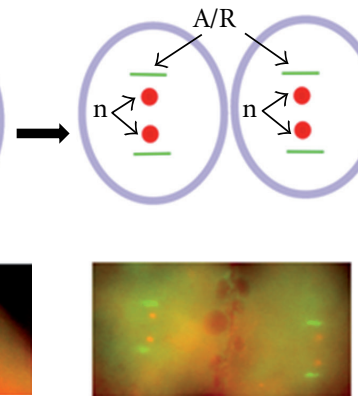

(b)

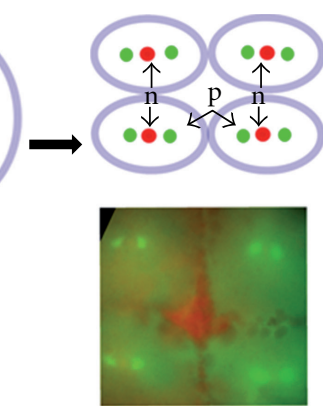

(c)
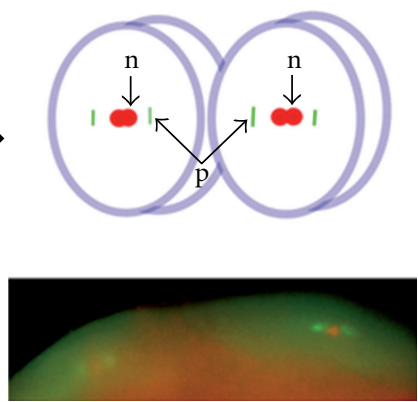

(d)

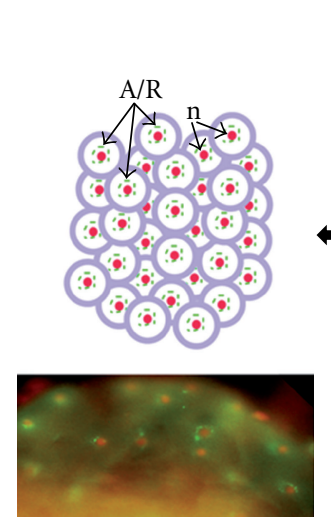

(h)

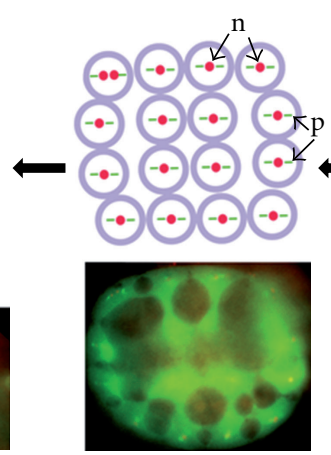

(g)

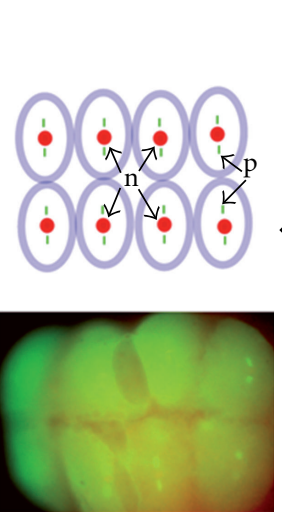

(f)

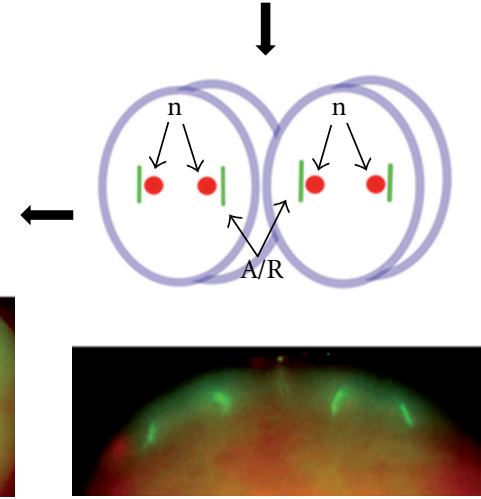

(e)

FIGURE 4: Summary of the alternating orientation of centrosomes and nuclei during zebrafish early embryogenesis using epifluorescence microscopy. Schematic cartoons of simplified nuclei ( $\mathrm{n}$; red dots) and curvilinear arc-shaped/ring (A/R) or punctate (p) centrosomes (green lines or dots, resp.) are depicted above each representative micrograph doubly labeled with DAPI (red) and GTU-88 (green). (a) First mitosis. (b) 2-cell stage with each cell in anaphase/telophase. (c) 4-cell stage with cells in prometaphase/metaphase. (d) 4-cell stage with each cell in early anaphase. (e) 4-cell stage with cells in telophase. (f) 8-cell stage, (g) 16-cell stage. (h) 32-cell stage.

and the vicinity of the condensed chromosomes aligned at both poles of the meiotic spindle at prometaphase I and metaphase I. At anaphase I and telophase I, $\gamma$-tubulin is detected between the separated chromosomes, while it is absent in the midbody. At the MII stage, $\gamma$-tubulin was again accumulated at the spindle poles. At an early stage of mouse fertilization, there was no $\gamma$-tubulin foci observed around the sperm chromatin or early male pronucleus [46]. At this stage, $\gamma$-tubulin is concentrated in the broad spindle poles of the meiotic spindle and at the distinct foci which form the centers of the cytoplasmic microtubule asters [47].

Besides Xenopus, oocytes lose centrioles during oogenesis in humans [48, 49], rhesus monkeys [50], cows [36], and many other mammals except rodents [35]. They also retain a stockpile of pericentriolar material (PCM), and microtubules are nucleated in the absence of centrioles in mammalian oocytes and early embryos [35]. This pool of $\gamma$-tubulin and centrosome components may play an important role in regulating MT organization during oocyte differentiation and animal-vegetal axis establishment [51]. In contrast, the spermatozoa lose most centrosomal proteins but retain centrioles during spermiogenesis [52]. The low abundance of endogenous $\gamma$-tubulin in most cells and the complexity of the seminiferous epithelial architecture make such evaluations in sperm cells extremely difficult [53]. In fact, we tried to visualize any possible centrosome structure by GTU- 88 staining in zebrafish sperm but none was detected (Liu and Lessman, unpublished data). So the turnover of $\gamma$-tubulin or other centrosomal proteins during zebrafish spermatogenesis is still unclear.

Centrosomes are assembled at fertilization, in which the centriole pair, inherited from the male gamete, binds nucleating components from the female gamete [18] and matures by acquiring maternally derived pericentriolar material components [52]. During fertilization, a functional centrosome is likely a composite structure blending both paternal and maternal centrosomal components. The mixing of the maternal and paternal chromosomes occurs at the first mitosis. In species such as sea urchin, C. elegans, Xenopus, and zebrafish, a diploid nucleus forms through the fusion of two haploid pronuclei before the first mitosis [32, 5456]. The process of pronuclear fusion occurs in zebrafish zygotes between 14 and 20 minutes postfertilization (mpf) [32]. In another cyprinid species, the common white sucker (Catostomus commersoni), an aster begins forming by $30 \mathrm{mpf}$ at one side of the fertilizing sperm cell within the ooplasm of the blastodisc. Formation of the male pronucleus, with growing aster, and an asterless female pronucleus is complete by $60 \mathrm{mpf}$ in this cool-water species $\left(16-18^{\circ} \mathrm{C}\right)$ [57].

In Caenorhabditis elegans, $\gamma$-tubulin is recruited around sperm centrioles after fertilization and accumulates massively at the centrosome during prophase of the first embryonic 
division. The centrosome undergoes striking morphological changes from metaphase to interphase. The compact structure visualized in metaphase changes into a spindle-like shape in anaphase before losing most of the $\gamma$-tubulin localization in telophase. In interphase, the $\gamma$-tubulin signal is similar in size to that of $\alpha$-tubulin, suggesting that most of the $\gamma$-tubulin protein left resides inside, or very near, the centriole. Concomitantly with these events, the number of microtubules nucleated at the centrosomes decreases considerably from telophase [25]. This "catastrophic" disassembly of $\gamma$-tubulin from the centrosome as cells exit mitosis has been reported in mammalian cells and is likely to be due to the release of most of the pericentriolar material into the cytoplasm [58]. The results suggest that the origin of mitotic spindle poles is associated with MTOCs near the condensed chromosomes and that spindle $\gamma$-tubulin is recruited from the cytoplasm during the process of spindle assembly.

In the mouse model, after fertilization, $\gamma$-tubulin foci in the egg cytoplasm and spindle poles were transformed to amorphous $\gamma$-tubulin and translocated from spindle poles to the area between the separating chromatids. When the pronuclei became enlarged and got close to each other, many $\gamma$-tubulin dots in the vicinity of the pronuclei were observed. The accumulation of $\gamma$-tubulin around the pronuclei may be responsible for extensive microtubule assembly in this region. It aggregated into some dots in interphase but was distributed on the mitotic spindle poles in early embryos [46]. The integrity of these $\gamma$-tubulin foci and their cytoplasmic location is maintained during drug- or cold-induced depolymerization of microtubules [59].

In unfertilized bovine oocytes, $\gamma$-tubulin was identified in the cytoplasm, mainly in the cortex and concentrated in the meiotic spindle. Following sperm penetration, $\gamma$ tubulin in the cytoplasm was recruited by a sperm component to reconstitute the zygotic centrosome. During pronuclear apposition, $\gamma$-tubulin was localized as spots at the spindle poles. $\gamma$-tubulin spots were also observed in blastomeres of embryos cleaved in vitro. In the later pronuclear stage, considerably less $\gamma$-tubulin and fewer microtubules were detected in the cytoplasm. At the mitotic metaphase of parthenotes, $\gamma$-tubulin was recruited to the condensed chromatin and concentrated in the spindle. In the absence of sperm components, the cell cycle-related assembly of $\gamma$ tubulin organizes microtubule nucleation for positioning the pronucleus and spindle protein of mitotic metaphase during the first cycle of bovine parthenotes [60].

When the Xenopus egg is fertilized, the centrosome is assembled from both paternal components (the centriole itself) and maternal components ( $\gamma$-tubulin; [61]). The latter are stored in the oocyte cytoplasm during oogenesis in sufficient quantities to contribute to the formation of thousands of centrosomes until the onset of massive zygotic gene expression, at the midblastula transition [41, 62]. Extracts prepared from embryos taken later in development actually cannot assemble centrosomes into sperm centrioles. Our current data suggest that the assembly of zebrafish early embryo may adopt the similar pathway as Xenopus.

In summary, the distribution of $\gamma$-tubulin in animal oocytes and early embryos is more complex than initially assumed. Although the mechanism of recruitment of $\gamma$ tubulin to the sperm component is currently unclear, it appears to be true that a model of blended zygotic centrosome composed of maternal constituents is introduced to a paternal template after insemination [63]. The present study revealed no centrosomal structures assembling after egg activation with water alone, suggesting the requirement of the sperm for centrosome assembly in zebrafish. Our data are consistent with the idea that maternal $\gamma$-tubulin is recruited around sperm centrioles after fertilization and accumulates at the centrosomes during the first few embryonic divisions forming a variety of $\gamma$-tubulin-containing structures including punctate foci, arc-like/rings, and linear arrays. The sperm centrosome is required to organize the radial array of microtubules, called the sperm aster, within the inseminated oocyte of nonrodent mammals. Thus, specific regulatory events must take place at fertilization to turn on the nucleating activity of the sperm centrosome.

3.2. $\gamma$-Tubulin and Cell Cycle Regulation in Oocytes and Embryos. The colocalization of $\gamma$-tubulin and phosphorylated MAP kinase with microtubule assembly in both control and taxol-treated pig oocytes suggests that emanation of microtubules from the chromosomes may be regulated/directed by microtubule-organizing material including $\gamma$-tubulin and phosphorylated MAP kinase in pig oocytes [64].

In newt embryos, cyclin $\mathrm{B} 1$ begins to accumulate in the nucleus during interphase in synchronous cleavage, and its greatest expression is in the centrosomes and the nucleus at prometaphase. The centrosomes of the principle sperm nucleus and the zygote nucleus have greater accumulation of both $\gamma$-tubulin and cyclin B1 compared with the centrosomes of the accessory sperm nuclei [65]. These data suggest that cyclin B1 may play roles in regulating $\gamma$-tubulin distribution in dividing embryos.

Establishment of polarity in C. elegans embryos is dependent on the centrosome. The sperm contributes a pair of centrioles to the egg, and these centrioles remain incapable of polarizing the cortex while the egg completes meiosis. Coincident with the establishment of polarity, the centrioles recruit centrosomal proteins, several of which are required for polarity, suggesting that temporal regulation of centrosome assembly may control the initiation of polarization. They found that cyclin E-Cdk2 controls the recruitment of centrosomal proteins specifically at the time of polarity establishment. Cyclin E is required for several examples of asymmetric cell division and fate determination in C. elegans and Drosophila [66].

The cycles of centriole and centrosome duplication are intimately coupled. Centrioles initiate duplication at the onset of $S$ phase in a process that requires Cdk2 (reviewed in [67]). At this time a generative disc, a precursor of the daughter centriole, forms at right angles to the proximal end of each mother centrioles. Daughter centrioles elongate as the cell cycle progresses, reaching full length by G2/M. During the G2/M transition, the centrosome also matures, accumulating $\gamma$-tubulin and other centrosomal components, and 
increasing in size and nucleating capacity (reviewed in [68]). $\mathrm{Cdk} 2$ is not required for normal centrosome duplication, maturation, and bipolar mitotic spindle formation in mouse embryonic fibroblast cell. In contrast, Cdk2 deficiency completely abrogates aberrant centrosome duplication induced by a viral oncogene. These results indicate that normal and abnormal centrosome duplications have significantly different requirements for Cdk2 activity and point to a role of $\mathrm{Cdk} 2$ in licensing centrosomes for aberrant duplication [69].

\section{Methods}

4.1. Zebrafish. Wild-type zebrafish were obtained from a local distributor and housed in a dedicated, temperature $\left(28^{\circ} \mathrm{C}\right)$ and photoperiod $(14 \mathrm{~L})$ controlled fish room in racks of food-quality plastic containers (6 and 12 1) with flowthrough dechlorinated water. Fish were fed 1-2 times daily a diet of TetraMin and dried brine shrimp flakes. This research was carried out under an IACUC approved protocol.

\subsection{Fully Grown Oocyte Collection. Gravid adult female} zebrafish were decapitated and the spinal cords pithed, the whole ovary was removed and placed in a Petri dish half filled with Cortland's solution $\left(\mathrm{NaCl} 7.25 \mathrm{~g}, \mathrm{CaCl}_{2}-2 \mathrm{H}_{2} \mathrm{O} 0.23 \mathrm{~g}\right.$, $\mathrm{KCl} 0.38 \mathrm{~g}, \mathrm{MgSO}_{4}-7 \mathrm{H}_{2} \mathrm{O} 0.23 \mathrm{~g}, \mathrm{NaHCO}_{3} 1.0 \mathrm{~g}$, penicillin $30 \mathrm{mg}$, and streptomycin $50 \mathrm{mg}$ made up to $1000 \mathrm{~mL}$ with double distilled water). Then the opaque fully grown oocytes were dissected from the ovary with watchmaker forceps and pooled into a $1.5 \mathrm{~mL}$ microcentrifuge tube.

4.3. Oocyte Maturation In Vitro. The opaque fully grown oocytes were separated from the others following the above procedure, pooled into a $60 \times 15 \mathrm{~mm}$ Petri dish with $10 \mathrm{~mL}$ fresh Cortland's solution with $1 \mu \mathrm{g} / \mathrm{mL} 17 \alpha$ - and $20 \beta$ dihydroxyprogesterone (DHP) dispensed in steroid vehicle (EtOH : propylene glycol, $1: 1$ ), and incubated at $28^{\circ} \mathrm{C}$ for $6 \mathrm{hr}$. Aliquots of these in vitro maturing oocytes were then collected at different time points and pooled separately into a $1.5 \mathrm{~mL}$ microcentrifuge tube. The oocytes were then fixed in freshly made $4 \%$ paraformaldehyde at $4^{\circ} \mathrm{C}$ overnight. On the following day, methanol was gradually added until they were brought to $100 \%$ methanol. Then the oocytes were stored in $100 \%$ methanol at $-20^{\circ} \mathrm{C}$ until used for the next step in the analysis.

4.4. Embryo Collection. Male and female fish were placed in separate compartments within spawning tanks, and the separation barriers were removed just prior to the desired mating time. This allowed collection of embryos at precise times after fertilization. Crosses were set up in the afternoon using 3 females and 4-5 males per breeding tank. After mating, males and females were separated again and allowed to rest for at least one week before the next cross. Embryos were collected next morning and were washed to remove any debris [70]. Standard fish water [31] supplemented with methylene blue (final concentration was 2 ppm) was used for the washes and maintained at $28^{\circ} \mathrm{C}$. Washed embryos were grouped according to the zebrafish stage tables [31] and collected into separate Eppendorf tubes. For further drug treatment, the embryos were grouped into Petri dishes and treated with different drugs. All the sample embryos were then fixed and dehydrated following the above procedures outlined for oocytes.

4.5. Antibody. Anti- $\gamma$-tubulin mouse monoclonal antibody GTU-88 (mouse IgG1 isotype; Sigma Chem. Co., St. Louis, MO) recognizes EEFATEGTDRKDVFFY located in position $38-53$ of the amino acid sequence of $\gamma$-tubulin near $\mathrm{N}$ terminal end. GTU-88 reacts well to zebrafish, [28, 32] human, bovine, dog, hamster, rat, mouse, chicken, and Xenopus $\gamma$-tubulin [71].

4.6. Immunofluorescence Microscopy. Before incubation in primary antibody, the fixed oocytes or embryos were rehydrated into $100 \%$ PBS in a graded series. The chorions of embryos were manually removed with watchmaker forceps after rehydration. Then the sample oocytes or embryos were incubated in 5\% nonfat milk (Carnation) in PBS solution for 4-6 hours at room temperature with gentle rotation. After the samples were washed several times with PBS, they were incubated with $1 \mathrm{~mL}$ 1: 200 primary antibody GTU-88 overnight at $4^{\circ} \mathrm{C}$. Then the oocytes or embryos were washed in PBS for 8 hours at room temperature, changing buffer at 1-hour intervals. The embryos were then incubated with $750 \mu \mathrm{L}$ 1: 200 secondary Alexa green-conjugated-anti-mouse antibody overnight at $4^{\circ} \mathrm{C}$. After the oocytes or embryos were washed several times with PBS (for 8 hours at room temperature, changing buffer at 1-hour intervals), the samples were incubated with $1 \mathrm{~mL} \mathrm{4}$ ',6-diamidino-2-phenylindole (DAPI) solution (final concentration is $2.5 \mu \mathrm{g} / \mathrm{mL}$ ) for 5 minutes to stain DNA, with gentle rotation at room temperature. Then the staining solution was decanted, and the embryos were washed with PBS for 8 hours to overnight at room temperature. The oocytes or embryos were then dehydrated gradually with $100 \%$ methanol, three or four changes over 60-90 minutes at room temperature. After the methanol was removed, the samples were cleared with Benzyl Benzoate : Benzyl alcohol $(\mathrm{BB}: \mathrm{BA}=2: 1)$ solution and mounted on slides in $\mathrm{BB}$ : BA solution. Then the oocytes or embryos were examined using either a Nikon laser scanning confocal microscope or Nikon eclipse 400 epifluorescence microscope. Digital RGB images captured with cameras mounted on the microscopes were processed (i.e., RGB split/merge), and reiterative deconvolution was performed on images to remove unfocused light using the ImageJ program (http://rsb.info.nih.gov/ij/). Generally, for epifluorescence images, 3 iterations were computed using the $2 \mathrm{D}$ deconvolution and diffraction limit point-spread-function (psf) ImageJ plug-ins available at http://www.optinav.com/imagej.html.

4.7. Confocal Microscopy. Embryos, oocytes, and eggs were examined on a Nikon C1 laser confocal microscope equipped with Nikon triple-laser scanning assembly using Argon (ex.488 nm), green helium-neon (ex.543 nm), and red 
helium-neon (ex.633 nm) lasers. Objectives of 10X and 20X were used. Some images presented are projections of up to three optical sections, providing increased depth of focus.

\section{Abbreviations}

MT: $\quad$ Microtubule

DHP: $\quad 17 \alpha-20 \beta$-dihydroxyprogesterone

MTOC: Microtubule organizing center

$\gamma$-TuRC: $\gamma$-Tubulin ring complex

DAPI: $\quad$ 4',6-Diamidino-2-phenylindole

GV: $\quad$ Germinal vesicle

Bl: $\quad$ Blastodisc.

\section{Conflict of Interests}

The authors have no financial or other conflict of interests regarding this work.

\section{Acknowledgments}

This work was supported in part by the Department of Biology, the University of Memphis Graduate School, a Faculty Research Grant from the University of Memphis, and the American Society for Cell Biology Minority Affairs Council. The authors thank the Integrated Microscopy Center, the University of Memphis, for their support in confocal microscopy analysis.

\section{References}

[1] F. Schnorrer, S. Luschnig, I. Koch, and C. Nüsslein-Volhard, " $\gamma$ tubulin37C and $\gamma$-tubulin ring complex protein 75 are essential for bicoid RNA localization during Drosophila oogenesis," Developmental Cell, vol. 3, no. 5, pp. 685-696, 2002.

[2] S. Choo, B. Heinrich, J. N. Betley, Z. Chen, and J. O. Deshler, "Evidence for common machinery utilized by the early and late RNA localization pathways in Xenopus oocytes," Developmental Biology, vol. 278, no. 1, pp. 103-117, 2005.

[3] S. Jesuthasan, "Furrow-associated microtubule arrays are required for the cohesion of zebrafish blastomeres following cytokinesis," Journal of Cell Science, vol. 111, no. 24, pp. 3695-3703, 1998.

[4] M. Wühr, N. D. Obholzer, S. G. Megason, H. W. Detrich, and T. J. Mitchison, "Live imaging of the cytoskeleton in early cleavagestage zebrafish embryos," Methods in Cell Biology, vol. 101, pp. $1-12,2011$

[5] L. Evans, T. Mitchison, and M. Kirschner, "Influence of the centrosome on the structure of nucleated microtubules.", Journal of Cell Biology, vol. 100, no. 4, pp. 1185-1191, 1985.

[6] M. Bornens, "Centrosome composition and microtubule anchoring mechanisms," Current Opinion in Cell Biology, vol. 14, no. 1, pp. 25-34, 2002.

[7] D. R. Kellogg, M. Mortiz, and B. M. Alberts, "The centrosome and cellular organization," Annual Review of Biochemistry, vol. 63, pp. 639-674, 1994.

[8] A. Kalt and M. Schliwa, "Molecular components of the centrosome," Trends in Cell Biology, vol. 3, no. 4, pp. 118-128, 1993.
[9] C. A. Lessman, "Centrosomes in the zebrafish (Danio rerio): a review including the related basal body," BMC Cilia, vol. 1, pp. 9-22, 2012.

[10] R. R. Gould and G. G. Borisy, "The pericentriolar material in Chinese hamster ovary cells nucleates microtubule formation," Journal of Cell Biology, vol. 73, no. 3, pp. 601-615, 1977.

[11] M. Moritz, M. B. Braunfeld, J. W. Sedat, B. Alberts, and D. A. Agard, "Microtubule nucleation by $\gamma$-tubulin-containing rings in the centrosome," Nature, vol. 378, no. 6557, pp. 638-640, 1995.

[12] K. Oegema, C. Wiese, O. C. Martin et al., "Characterization of two related Drosophila $\gamma$-tubulin complexes that differ in their ability to nucleate microtubules," Journal of Cell Biology, vol. 144, no. 4, pp. 721-733, 1999.

[13] R. N. Gunawardane, O. C. Martin, K. Cao et al., "Characterization and reconstitution of Drosophila $\gamma$-tubulin ring complex subunits," Journal of Cell Biology, vol. 151, no. 7, pp. 1513-1523, 2000.

[14] C. Wiese and Y. Zheng, " $\gamma$-tubulin complexes and their interaction with microtubule-organizing centers," Current Opinion in Structural Biology, vol. 9, no. 2, pp. 250-259, 1999.

[15] B. J. Soltys and G. G. Borisy, "Polymerization of tubulin in vivo: direct evidence for assembly onto microtubule ends and from centrosomes," Journal of Cell Biology, vol. 100, no. 5, pp. 1682-1689, 1985.

[16] C. E. Oakley and B. R. Oakley, "Identification of $\gamma$-tubulin, a new member of the tubulin superfamily encoded by mipA gene of Aspergillus nidulans," Nature, vol. 338, no. 6217, pp. 662-664, 1989.

[17] B. R. Oakley, C. E. Oakley, Y. Yoon, and M. K. Jung, “ $\gamma$-Tubulin is a component of the spindle pole body that is essential for microtubule function in aspergillus nidulans," Cell, vol. 61, no. 7, pp. 1289-1301, 1990.

[18] T. Stearns and M. Kirschner, "In vitro reconstitution of centrosome assembly and function: the central role of $\gamma$-tubulin," Cell, vol. 76, no. 4, pp. 623-637, 1994.

[19] T. Stearns, L. Evans, and M. Kirschner, " $\gamma$-tubulin is a highly conserved component of the centrosome," Cell, vol. 65, no. 5, pp. 825-836, 1991.

[20] Y. Zheng, M. K. Jung, and B. R. Oakley, " $\gamma$-tubulin is present in Drosophila melanogaster and homo sapiens and is associated with the centrosome," Cell, vol. 65, no. 5, pp. 817-823, 1991.

[21] S. J. Doxsey, P. Stein, L. Evans, P. D. Calarco, and M. Kirschner, "Pericentrin, a highly conserved centrosome protein involved in microtubule organization," Cell, vol. 76, no. 4, pp. 639-650, 1994.

[22] H. C. Joshi, "Microtubule organizing centers and $\gamma$-tubulin," Current Opinion in Cell Biology, vol. 6, pp. 54-62, 1994.

[23] M. Moritz, Y. Zheng, B. M. Alberts, and K. Oegema, "Recruitment of the $\gamma$-tubulin ring complex to Drosophila salt-stripped centrosome scaffolds," Journal of Cell Biology, vol. 142, no. 3, pp. 775-786, 1998.

[24] W. C. Zimmerman, J. Sillibourne, J. Rosa, and S. J. Doxsey, "Mitosis-specific anchoring of $\gamma$ tubulin complexes by pericentrin controls spindle organization and mitotic entry," Molecular Biology of the Cell, vol. 15, no. 8, pp. 3642-3657, 2004.

[25] Y. Bobinnec, M. Fukuda, and E. Nishida, "Identification and characterization of Caenorhabditis elegans $\gamma$-tubulin in dividing cells and differentiated tissues," Journal of Cell Science, vol. 113, no. 21, pp. 3747-3759, 2000. 
[26] I. Lajoie-Mazenc, Y. Tollon, C. Detraves et al., "Recruitment of antigenic $\gamma$-tubulin during mitosis in animal cells: presence of $\gamma$-tubulin in the mitotic spindle," Journal of Cell Science, vol. 107, no. 10, pp. 2825-2837, 1994.

[27] W. Driever, D. Stemple, A. Schier, and J. Solnica-Krezel, "Zebrafish: genetic tools for studying vertebrate development," Trends in Genetics, vol. 10, no. 5, pp. 152-159, 1994.

[28] J. Liu and C. A. Lessman, "Soluble tubulin complexes, $\gamma$ tubulin, and their changing distribution in the zebrafish (Danio rerio) ovary, oocyte and embryo," Comparative Biochemistry and Physiology B, vol. 147, no. 1, pp. 56-73, 2007.

[29] D. A. Kane, "Cell cycles and development in the embryonic zebrafish," Methods in Cell Biology, no. 59, pp. 11-26, 1999.

[30] D. A. Kane and C. B. Kimmel, "The zebrafish midblastula transition," Development, vol. 119, no. 2, pp. 447-456, 1993.

[31] M. Westerfield, The Zebrafish Book. A Guide For the Laboratory Use of Zebrafish (Danio Rerio), University of Oregon Press, Eugene, Ore, USA, 4th edition, 2004.

[32] M. P. S. Dekens, F. J. Pelegri, H. M. Maischein, and C. NüssleinVolhard, "The maternal-effect gene futile cycle is essential for pronuclear congression and mitotic spindle assembly in the zebrafish zygote," Development, vol. 130, no. 17, pp. 3907-3916, 2003.

[33] C. A. Lessman, R. Nathani, R. Uddin, J. Walker, and J. Liu, "Computer-aided meiotic maturation assay (CAMMA) of zebrafish (Danio rerio) oocytes in vitro," Molecular Reproduction and Development, vol. 74, no. 1, pp. 97-107, 2007.

[34] J. Liu and C. A. Lessman, "Changes of $\gamma$-tubulin expression and distribution in the zebrafish (Danio rerio) ovary, oocyte and embryo," Gene Expression Patterns, vol. 8, no. 4, pp. 237-247, 2008.

[35] G. Schatten, "The centrosome and its mode of inheritance: the reduction of the centrosome during gametogenesis and its restoration during fertilization," Developmental Biology, vol. 165, no. 2, pp. 299-335, 1994.

[36] C. S. Navara, N. L. First, and G. Schatten, "Microtubule organization in the cow during fertilization, polyspermy, parthenogenesis, and nuclear transfer: the role of the sperm aster," Developmental Biology, vol. 162, no. 1, pp. 29-40, 1994.

[37] B. J. Schnackenberg, A. Khodjakov, C. L. Rieder, and R. E. Palazzo, "The disassembly and reassembly of functional centrosomes in vitro," Proceedings of the National Academy of Sciences of the United States of America, vol. 95, no. 16, pp. 9295-9300, 1998.

[38] M. Kirkham, T. Müller-Reichert, K. Oegema, S. Grill, and A. A. Hyman, "SAS-4 is a C. elegans centriolar protein that controls centrosome size," Cell, vol. 112, no. 4, pp. 575-587, 2003.

[39] S. R. Scharf and J. C. Gerhart, "Determination of the dorsalventral axis in eggs of Xenopus laevis: complete rescue of uvimpaired eggs by oblique orientation before first cleavage.," Developmental Biology, vol. 79, no. 1, pp. 181-198, 1980.

[40] D. L. Gard, "Organization, nucleation, and acetylation of microtubules in Xenopus laevis oocytes: a study by confocal immunofluorescence microscopy," Developmental Biology, vol. 143, no. 2, pp. 346-362, 1991.

[41] D. L. Gard, S. Hafezi, T. Zhang, and S. J. Doxsey, "Centrosome duplication continues in cycloheximide-treated Xenopus blastulae in the absence of a detectable cell cycle," Journal of Cell Biology, vol. 110, no. 6, pp. 2033-2042, 1990.

[42] F. Verde, J. M. Berrez, C. Antony, and E. Karsenti, “Taxolinduced microtubule asters in mitotic extracts of Xenopus eggs: requirement for phosphorylated factors and cytoplasmic dynein," Journal of Cell Biology, vol. 112, no. 6, pp. 1177-1187, 1991.

[43] D. L. Gard, " $\gamma$-Tubulin is asymmetrically distributed in the cortex of Xenopus oocytes," Developmental Biology, vol. 161, no. 1, pp. 131-140, 1994.

[44] D. L. Gard, D. Affleck, and B. M. Error, "Microtubule organization, acetylation, and nucleation in Xenopus laevis oocytes: II. A developmental transition in microtubule organization during early diplotene," Developmental Biology, vol. 168, no. 1, pp. 189-201, 1995.

[45] X. Li, Y. Qin, S. Wilsher, and W. R. Allen, "Centrosome changes during meiosis in horse oocytes and first embryonic cell cycle organization following parthenogenesis, fertilization and nuclear transfer," Reproduction, vol. 131, no. 4, pp. 661-667, 2006.

[46] X. Q. Meng, H. Y. Fan, Z. S. Zhong et al., "Localization of $\gamma$ tubulin in mouse eggs during meiotic maturation, fertilization, and early embryonic development," Journal of Reproduction and Development, vol. 50, no. 1, pp. 97-105, 2004.

[47] M. J. Palacios, H. C. Joshi, C. Simerly, and G. Schatten, “ $\gamma$ Tubulin reorganization during mouse fertilization and early development," Journal of Cell Science, vol. 104, no. 2, pp. 383-389, 1993.

[48] C. Simerly, G. J. Wu, S. Zoran et al., “The paternal inheritance of the centrosome, the cell's microtubule-organizing center, in humans, and the implications for infertility," Nature Medicine, vol. 1, pp. 47-52, 1995.

[49] A. H. Sathananthan, S. S. Ratnam, S. C. Ng, J. J. Tarín, L. Gianaroli, and A. Trounson, "The sperm centriole: its inheritance, replication and perpetuation in early human embryos," Human Reproduction, vol. 11, no. 2, pp. 345-356, 1996.

[50] G. J. Wu, C. Simerly, S. S. Zoran, L. R. Funte, and G. Schatten, "Microtubule and chromatin dynamics during fertilization and early development in rhesus monkeys, and regulation by intracellular calcium ions," Biology of Reproduction, vol. 55, no. 2, pp. 260-270, 1996.

[51] D. L. Gard, " $\gamma$-Tubulin is asymmetrically distributed in the cortex of Xenopus oocytes," Developmental Biology, vol. 161, no. 1, pp. 131-140, 1994.

[52] G. Manandhar, H. Schatten, and P. Sutovsky, "Centrosome reduction during gametogenesis and its significance," Biology of Reproduction, vol. 72, no. 1, pp. 2-13, 2005.

[53] S. L. Fleming, P. R. Shank, and K. Boekelheide, " $\gamma$-tubulin overexpression in sertoli cells in vivo: I. Localization to sites of spermatid head attachment and alterations in sertoli cell microtubule distribution," Biology of Reproduction, vol. 69, no. 1, pp. 310-321, 2003.

[54] F. J. Longo and E. Anderson, "The fine structure of pronuclear development and fusion in the sea urchin, Arbacia punctulata., Journal of Cell Biology, vol. 39, no. 2, pp. 339-368, 1968.

[55] G. A. Ubbels, K. Hara, C. H. Koster, and M. W. Kirschner, "Evidence for a functional role of the cytoskeleton in determination of the dorsoventral axis in Xenopus laevis eggs," Journal of Embryology and Experimental Morphology, vol. 77, pp. 15-37, 1983.

[56] S. Strome and W. B. Wood, "Generation of asymmetry and segregation of germ-line in early C. elegans embryos," Cell, vol. 35, no. 1, pp. 15-25, 1983.

[57] C. A. Lessman and C. W. Huver, "Quantification of fertilization-induced gamete changes and sperm entry without egg 
activation in a teleost egg," Developmental Biology, vol. 84, no. 1, pp. 218-224, 1981.

[58] J. B. Dictenberg, W. Zimmerman, C. A. Sparks et al., "Pericentrin and $\gamma$-tubulin form a protein complex and are organized into a novel lattice at the centrosome," Journal of Cell Biology, vol. 141, no. 1, pp. 163-174, 1998.

[59] C. Gueth-Hallonet, C. Antony, J. Aghion et al., " $\gamma$-Tubulin is present in acentriolar MTOCs during early mouse development," Journal of Cell Science, vol. 105, no. 1, pp. 157-166, 1993.

[60] M. R. Shin and N. H. Kim, "Maternal $\gamma(\gamma)$-tubulin is involved in microtubule reorganization during bovine fertilization and parthenogenesis," Molecular Reproduction and Development, vol. 64, no. 4, pp. 438-445, 2003.

[61] M. A. Felix, C. Antony, M. Wright, and B. Maro, "Centrosome assembly in vitro: role of $\gamma$-tubulin recruitment in Xenopus sperm aster formation," Journal of Cell Biology, vol. 124, no. 1-2, pp. 19-31, 1994.

[62] J. Newport and M. Kirschner, "A major developmental transition in early Xenopus embryos: I. Characterization and timing of cellular changes at the midblastula stage," Cell, vol. 30, no. 3 , pp. 675-686, 1982.

[63] C. Simerly, S. S. Zoran, C. Payne et al., "Biparental inheritance of $\gamma$-tubulin during human fertilization: molecular reconstitution of functional zygotic centrosomes in inseminated human oocytes and in cell-free extracts nucleated by human sperm," Molecular Biology of the Cell, vol. 10, no. 9, pp. 2955-2969, 1999.

[64] Q. Y. Sun, L. Lai, G. M. Wu et al., "Microtubule assembly after treatment of pig oocytes with taxol: correlation with chromosomes, $\gamma$-tubulin, and MAP kinase," Molecular Reproduction and Development, vol. 60, no. 4, pp. 481-490, 2001.

[65] Y. Iwao, T. Murakawa, J. Yamaguchi, and M. Yamashita, "Localization of $\gamma$-tubulin and cyclin B during early cleavage in physiologically polyspermic newt eggs," Development Growth and Differentiation, vol. 44, no. 6, pp. 489-499, 2002.

[66] C. R. Cowan and A. A. Hyman, "Cyclin E-Cdk2 temporally regulates centrosome assembly and establishment of polarity in Caenorhabditis elegans embryos," Nature Cell Biology, vol. 8, no. 12, pp. 1441-1447, 2006.

[67] A. M. Fry, P. Descombes, C. Twomey, R. Bacchieri, and E. A. Nigg, "The NIMA-related kinase X-Nek2B is required for efficient assembly of the zygotic centrosome in Xenopus laevis," Journal of Cell Science, vol. 113, no. 11, pp. 1973-1984, 2000.

[68] R. E. Palazzo, J. M. Vogel, B. J. Schnackenberg, D. R. Hull, and X. Wu, "Centrosome maturation," Current Topics in Developmental Biology, vol. 49, pp. 449-470, 1999.

[69] A. Duensing, Y. Liu, M. Tseng, M. Malumbres, M. Barbacid, and S. Duensing, "Cyclin-dependent kinase 2 is dispensable for normal centrosome duplication but required for oncogeneinduced centrosome overduplication," Oncogene, vol. 25, no. 20, pp. 2943-2949, 2006.

[70] Y. Grinblat, M. E. Lane, C. Sagerström, and H. Sive, "Analysis of zebrafish development using explant culture assays," Methods in Cell Biology, no. 59, pp. 127-156, 1999.

[71] B. R. Oakley, " $\gamma$-Tubulin: the microtubule organizer?" Trends in Cell Biology, vol. 2, no. 1, pp. 1-5, 1992.

[72] K. Selman, R. A. Wallace, A. Sarka, and X. Qi, "Stages of oocyte development in the zebrafish, Brachydanio rerio," Journal of Morphology, vol. 218, no. 2, pp. 203-224, 1993. 

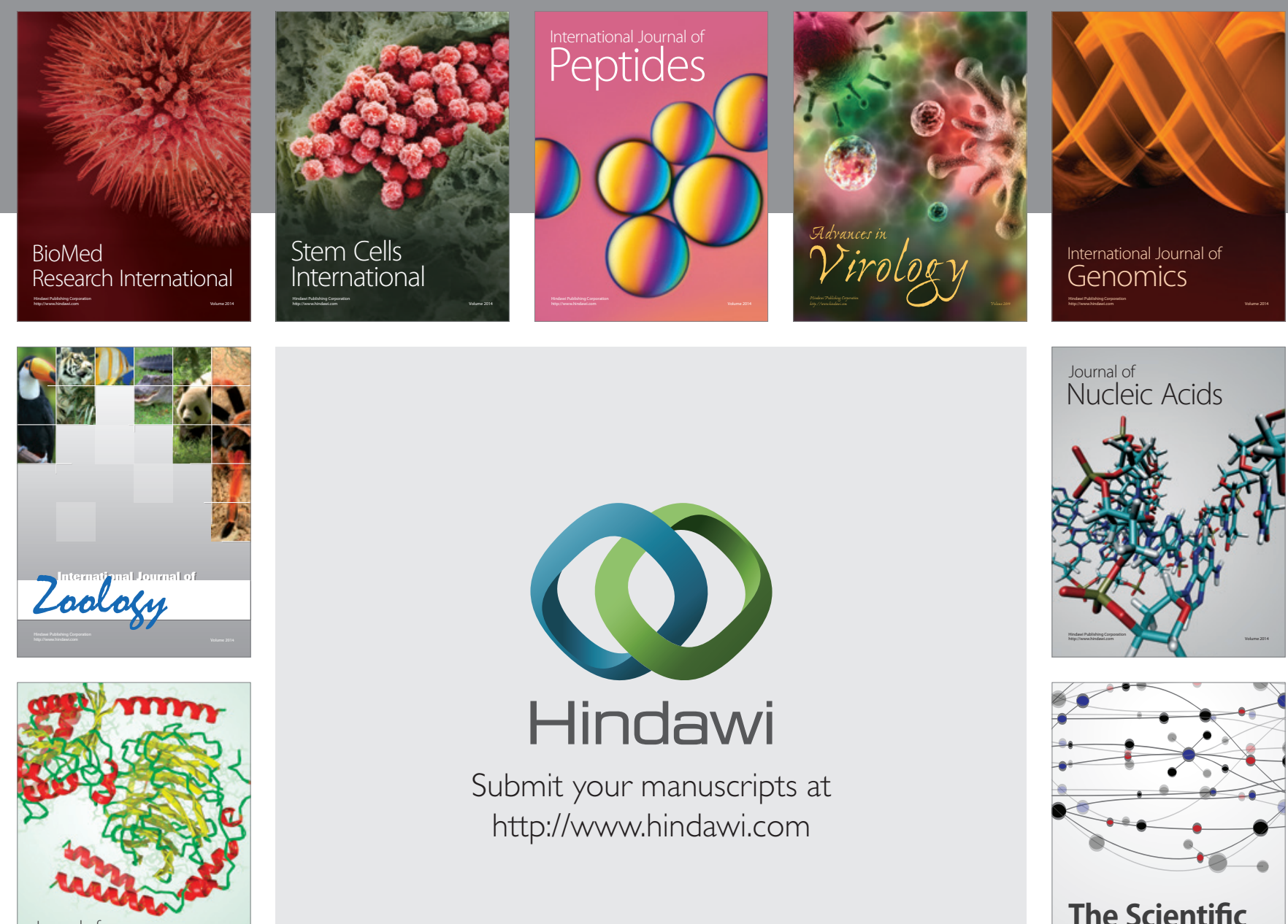

Submit your manuscripts at

http://www.hindawi.com

Journal of
Signal Transduction
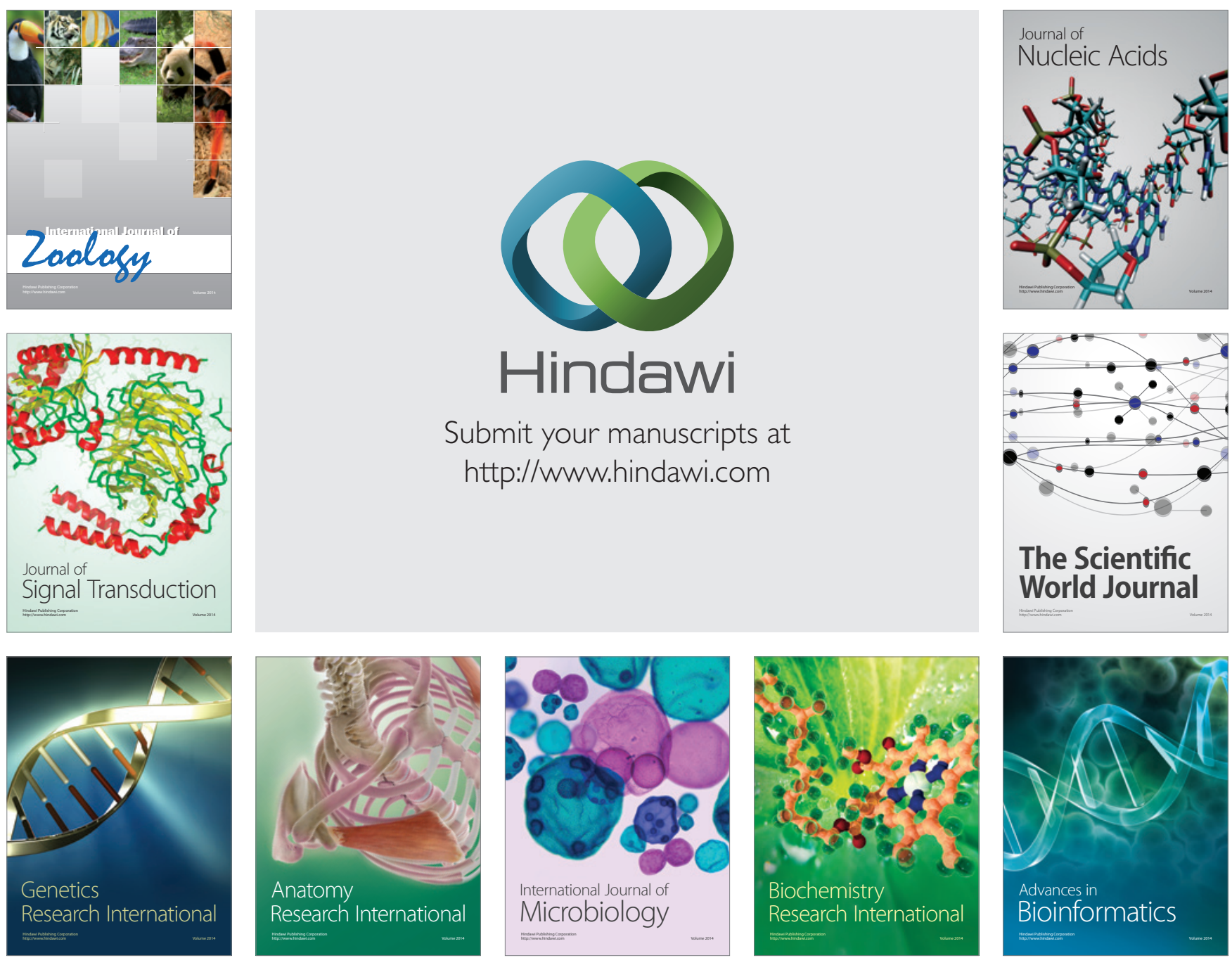

The Scientific World Journal
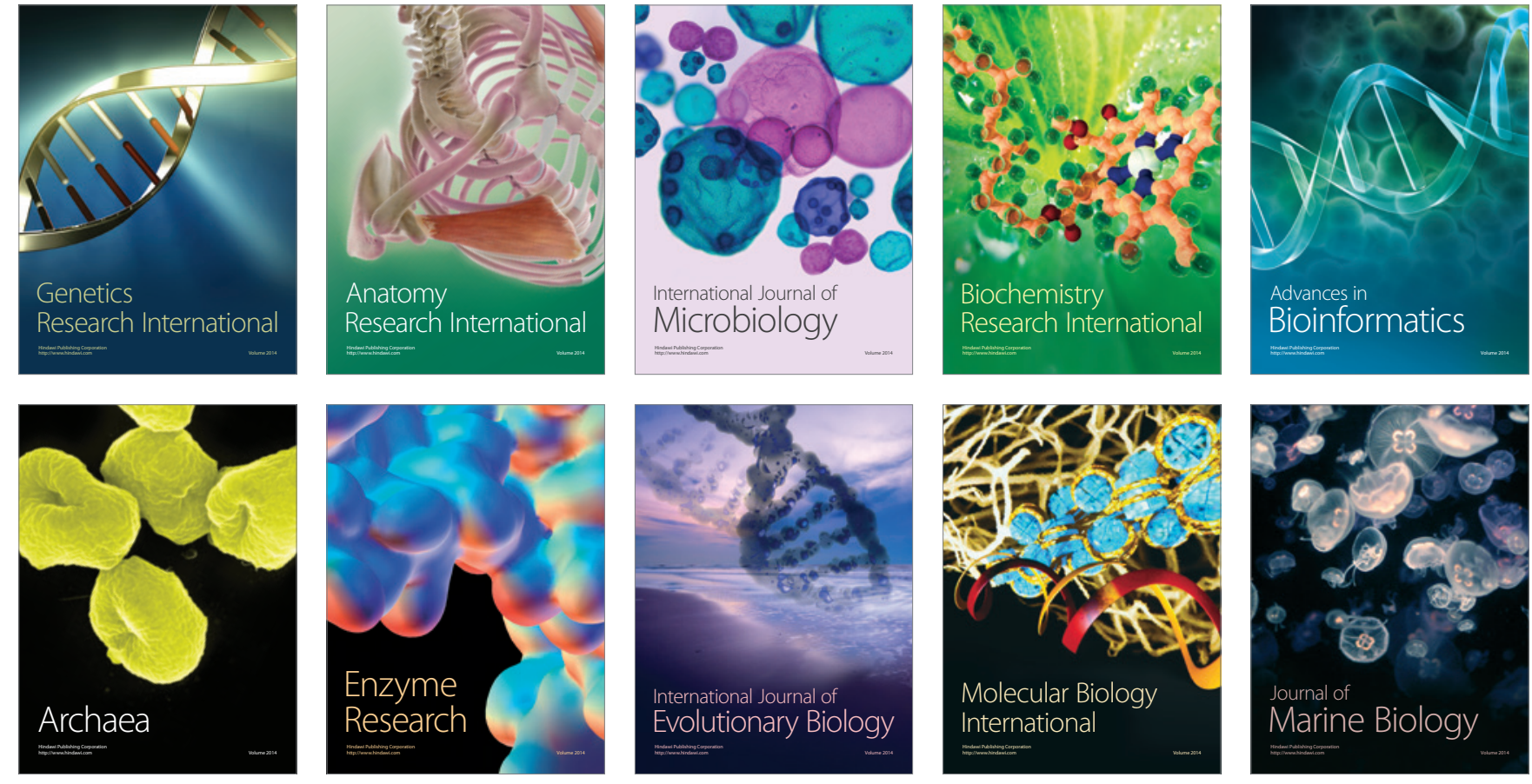